\title{
Exploring the Solution Space of Beaconing in VANETs
}

\author{
Martijn van Eenennaam, Wouter Klein Wolterink, Georgios Karagiannis, Geert Heijenk \\ Department of Computer Science, University of Twente, The Netherlands \\ \{e.m.vaneenennaam, w.kleinwolterink, g.karagiannis, geert.heijenk\}@utwente.nl
}

\begin{abstract}
Vehicular networking is an enabling technology for Intelligent Transportation Systems (ITS). Different types of vehicular traffic applications are currently being investigated. In this paper we briefly introduce the communication requirements of a Co-operative Adaptive Cruise Control (C-ACC) vehicular traffic efficiency application. Furthermore, we propose a Channel Busy Time model to evaluate the solution space of a vehicular beaconing system designed to communicate information both vital and sufficient for vehicular traffic applications and in particular for C-ACC. We identify that the solution space is three-dimensional. These dimensions being based on the number of nodes (or vehicles), the beacon generation rate of the nodes and the size (or duration) of a beacon message.

Based on the Channel Busy Time model we derive boundaries and ranges of parameters within which the beaconing system can be adapted to meet the requirements of the $\mathrm{C}$-ACC application.
\end{abstract}

\section{INTRODUCTION}

Vehicular networking can be considered as one of the most important enabling technologies for Intelligent Transportation Systems (ITS). Vehicular networking aims to support various types of traffic applications with varying goals, such as infotainment, traffic efficiency \& management, and traffic safety. Traffic safety applications are those that are primarily applied to decrease the probability of traffic accidents and loss of life. Traffic efficiency \& management applications are focusing on improving the vehicle traffic flow, traffic coordination, and driver assistance. Infotainment applications are, for instance, media downloading, instant messaging etc.

In this paper we consider a Co-operative Adaptive Cruise Control (C-ACC) application, which is a traffic efficiency application, described in detail in Sec. II. C-ACC relies on accurate and timely situational awareness to perform its task: aid in the longitudinal control of the vehicle like the traditional Cruise Control, but improved with enhanced situational awareness based on information communicated by the vehicles in front. We reason this awareness can be built using a beaconing approach. These beacons contain information such as speed, acceleration and position. Beaconing is quite an ambiguous term and is used in various contexts:

1) An access point periodically announces its presence by broadcasting a beacon frame [1]. This frame contains information necessary for a wireless node to associate with the access point. In Distributed Coordination Function (DCF) mode, beacons can be used in a distributed manner for timing synchronization (TSF) [1];

2) Some MANET (Mobile Ad-hoc Network) ad-hoc routing / forwarding protocols rely on one or two-hop neigh-

This work is supported by the Dutch Senter Novem/HTAS (High Tech Automotive Systems) Project Connect\&Drive, Project no. HTASD08002 bor knowledge. This knowledge can be gathered either instantaneously when needed, or a priori by maintaining neighbor knowledge by the proactive exchange of small presence messages called beacons;

3) In a VANET (Vehicular Ad-hoc Network) scenario, every node periodically transmits short status messages to create a distributed awareness. The information contained in these beacons covers the network topology as well as the operational status of the vehicle. Within this scenario, beacons can be disseminated on a one- or multi-hop basis.

Beaconing of type (1) is applied to provide synchronization and to possibly exchange MAC (Medium Access Control) parameters such as frequency hopping parameters. Beaconing of type (2) is used by the network layer and type (3) by the application layer. In the VANET context, beaconing of type (3) is often considered, while some work evaluates beaconing of type (2) [2]. In this paper we assume that beaconing of type (3) is used to disseminate C-ACC traffic information.

Due to the fact that beacons may be sent several times per second and vehicular density can vary greatly (and become large during traffic jams), it is expected that the channel may become congested. An important challenge that is considered in this paper is to explore the beaconing solution boundaries, e.g. what is the available capacity and what knobs can be turned to design a beaconing scheme to meet C-ACC application requirements over a wide range of situations.

The research questions answered by this paper are:

1) What are the communication requirements of the beaconing-based C-ACC application?

2) What are the boundaries of the beaconing solution space?

3) Can the foreseen beaconing-based C-ACC application be projected within these beaconing solution boundaries?

The remainder of this paper is organized as follows. In Sec. II, background information and related work with respect to vehicular networking and ITS applications is presented. Sec. III describes the beaconing requirements imposed by C-ACC application and answers research question (1). The exploration of the beaconing solution boundaries is described in Sec. $\mathrm{V}$. This section answers research question (2) by means of a model, which is introduced in Sec. IV. Sec. VI answers research question (3) by evaluating the model against the CACC communication requirements. Finally, Sec. VII concludes and an outlook on possible future activities is given. 


\section{BACKGROUND AND RELATED WORK}

Within the world of vehicular networking numerous standardization bodies (e.g., ETSI [3], ISO [4]), stakeholders (including vehicle manufacturers, telecom operators, and $\mathrm{R} \& \mathrm{D}$ institutes), consortia (e.g., C2C-CC [5]) and (inter)national projects exist. As we do not have the space here to give a decent description of all parties involved or the ways in which they (co)operate, we refer the reader to [6] for a thorough overview and only mention the involved bodies that are relevant to our paper.

In 2008 COMeSafety [7], a major European project whose goal basically is to consolidate input from all involved parties into a single supported framework, published a baseline for a European ITS communication architecture. This architecture includes a.o. involved entities, technologies used and a network level communication protocol called the European ITS VANET Protocol (EIVP). We will refer back to the EIVP later on, for now Fig. 1 shows the main entities used in the COMeSafety architecture, as initially proposed by $\mathrm{C} 2 \mathrm{C}-\mathrm{CC}$ in [5].

Application Unit $(A U)$ : a processing feature that is located in a vehicle and is able to run traffic applications and use the communication capabilities provided by an OBU.

On Board Unit $(O B U)$ : processing and communication feature that is located in a vehicle and is able to provide an application runtime environment, positioning, security and communication functions and interfaces to other vehicles. OBUs can communicate with other OBUs and RSUs using Ad-hoc communication means. The wireless technology applied for Ad-hoc communication is the IEEE 802.11p [8] technology. OBUs can also communicate with entities located in the Infrastructure domain using other types of wireless technologies, such as IEEE $802.11 \mathrm{a} / \mathrm{b} / \mathrm{g}$ and UMTS (Universal Mobile Telecommunications System), using Hot Spots (HS) or base stations, respectively.

Road Side Unit (RSU): equipment located on fixed locations along highways, at intersections, and other types of locations where timely communications with vehicles are needed. RSUs can communicate with OBUs using Ad-hoc communication means. Moreover, RSUs can communicate with other RSUs either using the Ad-hoc communication means or via a Gateway (GW). GW is also used to interconnect RSUs with entities located in the Infrastructure domain.

Day by day the vehicle traffic density on the roads of most industrialized countries keeps increasing. As vehicle traffic density increases, so does the traffic congestion. This has a significant negative effect on travel time, traffic safety, air pollution, and energy consumption. Currently, a possible solution to this problem is to use the Adaptive Cruise Control (ACC) concept. ACC was initially developed to increase user comfort, but research activities have shown that ACC could indeed have a positive impact on traffic safety and efficiency [9]. By extending the Cruise Control system with a radar sensor, ACC allows a vehicle to maintain a preset speed, as well as to adapt its speed to the speed of its predecessor. In this case, the vehicle accelerates when the preceding

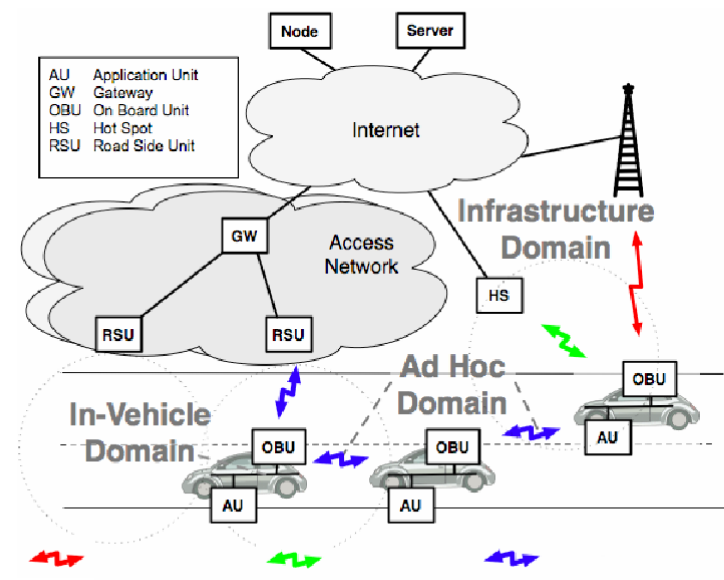

Figure 1: C2C-CC architecture, from [5]

vehicle is increasing its speed and it slows down when it is approaching a vehicle that is driving with a lower speed than its own. An enhancement on the ACC concept is the Co-operative ACC (C-ACC), where the OBU in a vehicle is using a communication medium to communicate with OBUs in other vehicles or RSUs. During this communication, a vehicle can obtain the necessary preceding vehicle dynamics information and general traffic information ahead, such as speed, acceleration, and position of other vehicles. In this paper, we denote this communicated information as C-ACC traffic information. The C-ACC traffic information can be used to enhance the performance of the current ACC systems. It is expected that C-ACC will increase vehicle traffic efficiency and traffic stability [9], [10]. C-ACC can be applied in traffic applications such as co-operative following [10], or vehicle platooning [11], [12].

The co-operative-following traffic application typically uses the information provided by the vehicular communication network in combination with longitudinal control. This allows for anticipation to emerging shock waves, with the goal to improve traffic safety and traffic efficiency. Key is that the system is completely ad hoc.

The research activities in the area of vehicle platooning are typically assuming that vehicles can use Automated Highway Systems (AHS) [13], where all vehicles using a road lane can communicate with each other either directly and/or via the road infrastructure using RSUs, implying some infrastructure.

Co-operative-following can have the same externallyobservable effects as vehicle platooning, and as such the difference is mainly in the implementation. Both approaches can shift the vehicle control tasks from the driver to the vehicle [14], [15]. This includes smooth following, merging, and lane changing maneuvers. By using co-operative following, the headway distance between vehicles within the platoon can be kept small, increasing the traffic capacity and traffic efficiency. However, the disturbances produced by vehicle acceleration or deceleration must not exceed a maximum value [16]. Vehicle platooning has been investigated in many papers, such as the PATH framework [17], [18], the Japanese Dolphin framework 
[19], the Auto21 Collaborative Driving System (CDS) [20], [21], and the framework in [22].

Not many references are specifying network performance requirements associated with vehicular traffic applications. The Vehicular Safety Communications projct (VSC) [23] is the main reference that describes performance requirements associated with vehicular traffic safety applications. [24] specifies performance requirements that are associated with C-ACC based traffic applications. The authors of this paper could not find any other related work than [24] that derived such performance requirements based on the input needed by the C-ACC automatic control unit.

\section{BEACONING REQUIREMENTS IMPOSED BY C-ACC}

This section discusses the requirements that are imposed by co-operative following applications such as $\mathrm{C}-\mathrm{ACC}$ on the communication medium and in particular on beaconing. In order to discuss these requirements, the results derived by the Dutch Connect \& Drive (C\&D) [25] project on vehicle platooning will be presented. In addition to this, this section discusses how these beaconing requirements differ from the requirements imposed by other traffic applications, such as traffic safety applications identified in [23].

The goal of C\&D is to investigate, design and implement a C-ACC system. To this end WiFi technology (i.e. IEEE 802.11 ) is used for the communication between vehicles and infrastructure. The targets of C-ACC are: (1) improve the capacity of the road infrastructure, (2) improve traffic safety and efficiency and (3) reduce the emission of vehicles. The C-ACC attacks traffic congestion at its roots, which are the limitations of the human vehicle control in reaction and visual field. In this way, the C-ACC system actively avoids and subdues congestion. The $\mathrm{C} \& \mathrm{D}$ project considers the vehicular networking architecture developed by $\mathrm{C} 2 \mathrm{C}-\mathrm{CC}$.

The requirements on beaconing derived by the $C \& D$ project are specified in [24]. The main differences between the beaconing requirements imposed by co-operative-following applications and traffic safety applications as defined in [23], are the following. In typical traffic safety applications the traffic application information according to [24] has to: be periodically generated by each vehicle, with a typical frequency of $10 \mathrm{~Hz}$; be disseminated with an allowable latency of 100 ms; be transmitted upstream by each vehicle and disseminated to all vehicles in the one hop communication range, typically up to $300 \mathrm{~m}$.

In our C-ACC application almost all of the required beacon information is typical to any traffic safety/efficiency application, such as vehicle position, -speed, -acceleration, dimensions, -identifier, etc. Besides this some (fewer than 30 bytes) room is needed for specific application commands. This information has to [24]: be periodically generated by each vehicle, with a typical frequency of $25 \mathrm{~Hz}$; the generation rate could be decreased if necessary, but it should not go below $10 \mathrm{~Hz}$; be disseminated with an allowable latency of $200 \mathrm{~ms}$; the C-ACC traffic information should reach at least $200 \mathrm{~m}$ or 15 vehicles per lane in the upstream direction.
The information should be disseminated one hop, and if needed beyond one hop, i.e., multi-hop beaconing. The required information to be disseminated is typical to any safety application and is fully covered by COMeSafety's EIVP Cooperative Awareness Message (CAM) [7] which has been chosen as the beacon message format. The purpose of the CAM is to provide both awareness of nearby vehicles and additional information for safety applications, making it ideally suited for our C-ACC application. Although the EIVP standard (including the CAM) is not yet final, we estimate the size of such a beacon (based on [7]) around 80 bytes, with room for additional payload. This excludes any security overhead, a topic which is still a subject of ongoing research and has been left open by COMeSafety. In [26] however, it is estimated that the required security overhead for such an application (i.e., certificate and signature) has a maximum length of 300 bytes. Based on this we assume the length of one C-ACC traffic information message to be approximately 400 bytes (including the additional application-specific data).

The CALM M5 specification [27] and IEEE 802.11 specification [1] specify that the maximum payload length of an IEEE 802.11 frame is 2304 bytes. The IEEE 1609 specification [28] specifies that the maximum size of a Wave Short Message Protocol (WSMP) message is 1400 bytes. In this paper we also assume that the maximum payload length of an IEEE 802.11p frame is 1400 bytes.

\section{Modelling the Beaconing Solution Space}

In order to explore the beaconing solution space we derive a channel utilization model, which consists of three main dimensions. These dimensions are based on the number of nodes, i.e., vehicles, the beacon generation rate of these nodes and the size or duration of the beacon message. Furthermore, we reason that the beacon reception probability is related to the channel utilization. The beacon reception probability, i.e., $P_{s}$, is the probability that a transmitted beacon can be successfully received by a vehicle.

For every vehicle, consider that the rate at which beacons from a certain vehicle are received $\left(\lambda_{r}\right)$ is a function of the beacons generated $\left(\lambda_{g}\right)$ and the probability that a beacon will be received: $\lambda_{r}=\lambda_{g} \cdot P_{s}$.

\section{A. Model Assumptions}

This section specifies all assumptions that have been made for the analytical and probabilistic models described in the next two sections. Unless specified otherwise all assumptions have also been made in [29] and [30], research activities that also explore the boundaries of the beacon solution space.

a) Topology: We assume a straight road with $l$ lanes. All vehicles are similar and are uniformly distributed on the road to achieve a certain density $(\rho)$ in vehicles per kilometer per lane. We abstract from mobility under the assumption that communication happens on a much smaller timescale than mobility and traffic can henceforth be considered static. In graphical form, this looks as shown in Fig. 2. 


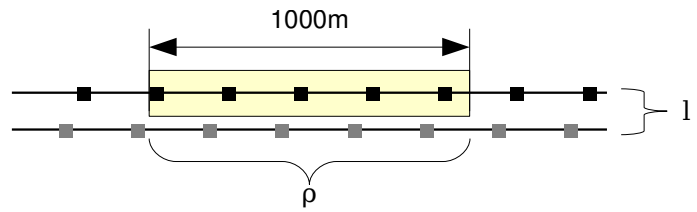

Figure 2: Node topology

b) Communication: All vehicles can communicate using an IEEE 802.11p MAC and PHY, the wireless channel is modeled using the Friis formula [31] with pathloss exponent $\alpha$. We consider omnidirectional antenna patterns, symmetric links, and a constant Interference Range (IR), Carrier Sense range (CS) and Communication Range (CR). The IR is the range up to which a nodes transmission will interfere with ongoing transmission, i.e., acts as noise. The CS represents the range up to which other nodes may sense an ongoing transmission (i.e., they sense that the medium is busy), but they may not necessarily be able to successfully receive the transmission. The CR then is defined as the range up to which a transmission is successfully received. Typically these ranges are dynamic in size and have overlapping sizes $(\mathrm{CR} ; \mathrm{CS} ;$ IR), for the sake of our model however they are constant and equal in size (i.e., $\mathrm{CR}=\mathrm{CS}=\mathrm{IR}$ ). For simplicity we assume unity antenna gain and system loss $\left(G_{t}=G_{r}=L=1\right)$.

In this paper we limit the analysis to a data rate of $3 \mathrm{Mbps}$, the lowest rate defined in $802.11 \mathrm{p}$, because this modulation scheme is the most robust to interference and noise [32]. We model channel busy time, e.g. when there is energy on the channel. Whether this energy is decoded as a successful message or as noise is discussed later. We consider our beaconing application in isolation, there are no other applications generating traffic or interference. Beacons only travel one-hop, they are not rebroadcast. Furthermore, we do not consider periodic channel switching as proposed for WAVE [33], we assume a node always listens to the medium.

\section{B. Channel Load}

We propose a simple expression for channel busy time based on [34]. This expression models the normalized channel load. Note that this model holds when transmissions do not overlap (there are no collisions). Under ideal channel conditions, the channel load can go up to 1 without collisions (i.e. $P_{s}=1$ ). Therefore this expression can be used to obtain an upperbound for the maximum load supported by the medium. We introduce the normalized channel load as follows:

$$
\mu=n \cdot \lambda_{g} \cdot T_{s}
$$

Where $n$ - the number of vehicles in CS range, $\lambda_{g}$ - the beacon generation rate, and $T_{s}$ - the duration of one beacon. We propose means to further detail these three factors, and identify tunable parameters to control the network load.

Measuring network load is important in order to be able to influence the generation of load by the nodes, a practise known as congestion control [34]. This is because the network functions optimally within a certain region of network load. Once the load becomes too high, performance rapidly drops.

The three factors which constitute the utilization are identified in [34] as parameters for a generic congestion control framework. We evaluate these parameters for one specific application, beaconing for a C-ACC system.

1) Number of nodes: The number of nodes $n$ is defined by the number of nodes within the CS range, these nodes share the channel. As such, ideally every node observes the channel in the same state.

The number of nodes has a strong relation with throughput, delay and delivery ratio [29], [35], [36]. Generally, more nodes results in a higher network load as modelled by the $n$ in Eq. (1). The number of nodes is modelled as follows:

$$
n=2 C S \cdot \rho \cdot l
$$

The CS is assumed to be omnidiretional (hence the factor 2 for our straight road). The traffic density is expressed by $\rho$ and is measured in vehicles per kilometer per lane. $l$ denotes the number of lanes of the road. CS can be defined by the Free Space model $^{2}[31]$ :

$$
C S=\left(\frac{P_{t} G_{t} G_{r} \lambda^{2}}{P_{r} 16 \pi^{2} L}\right)^{\frac{1}{\alpha}}
$$

Here $P_{t}$ and $P_{r}$ are the transmit power and receive threshold (i.e. the sensitivity), respectively. $G_{t}$ and $G_{r}$ are transmitter and receiver antenna gain, $\lambda$ is the wavelength. The factor $L$ models system loss and $\alpha$ the propagation pathloss

We assume that $G_{t}=G_{r}=1$ (all vehicles are equal), $L=1, \alpha=[2,5]$. These are common assumptions.

From Eq. (3) it becomes evident the following are means to throttle $n$ in Eq. (1):

$P_{t} \quad$ By increasing the transmission power, the transmission range increases. As such, for a given $\rho$ the number of nodes $n$ increases. Control of $P_{t}$ is applied in work by Torrent-Moreno et al. [32], [37] and Yang et al. [30].

$P_{r} \quad$ To derive CS, $P_{r}$ is set to the receive threshold (i.e. the minimum level at which a signal can be detected). This is hardware-dependent and related to the data rate (or actually, the robustness of the modulation scheme used). Generally, a higher data rate has a higher receive threshold and, as a result, a smaller CS than a low data rate for equal $P_{t}$.

2) Beacon Generation Rate: The beacon generation rate $\lambda_{g}$ determines how often per second a node generates a beacon. This has a direct effect on $\mu$ in Eq. (1).

Often beacons are assumed to be generated at fixed periodic rates (e.g. $10 \mathrm{~Hz}$ ). We reason that any application relying on beacon information should take into account that, due to the nature of radio propagation, some degree of messages may not arrive and the application should henceforth have the property

\footnotetext{
${ }^{2}$ note that a more sophisticated propagation model such as Nakagami, parameterized with values found during field tests, could be used.
} 
of gracefull degradation. In line with this reasoning, $\lambda_{g}$ can be lowered in case of congestion on the medium, since this increases the probability of correct reception.

Beacon generation can be modelled using a deterministic distribution or drawn from a certain random distribution (e.g. Normal with mean $\lambda_{g}$ and some variance). The exact implementation of beacon rate adaptation is still an open field within VANETs [34]. It is the aim of this paper to shed light on the possiblities; actual beacon rate adaptation protocols will be published in future work.

3) Beacon Duration: The time a beacon message is on the channel is modelled as follows:

$$
T_{s}=T_{h}+\frac{S}{R}
$$

where:

$T_{h}$ time to transmit the Preamble + Physical Layer Convergence Procedure (PLCP) header

$S \quad$ the size of a beacon message in bits

$R$ the data rate in bits/s

In Eq. (4), $T_{h}$ and $\frac{S}{R}$ model the time the signal is on the medium. This offers two means to influence $\mu$ in Eq. (1):

- By efficiently coding the beacon, the packet can be kept small. This is trivial for one beacon message per frame, but there may be schemes where information received from multiple nodes is repeated in a beacon (i.e. a composite beacon). In such case these beacons may be compressed. Härri et al. [38] propose a technique for coordinate compression with 70 per cent gain. Kargl et al. propose means to deal with security overhead [39] both in payload size and computation. It is imperative that a future implementation codes beacons as efficiently as possible.

$\mathrm{R} \quad-T_{s}$ depends greatly on the data rate. As $\mathrm{R}$ increases, $T_{s}$ decreases. It seems tempting to increase the data rate $(802.11 \mathrm{p}$ supports a maximum data rate of 27 Mbps) but it should be mentioned that larger $\mathrm{R}$ may have a negative impact on the probability of correct reception, as transmissions at higher data rates are more prone to interference. Data rate should, however, be considered as an important parameter in controlling the congestion on the channel because of its direct relation to $T_{s}$.

\section{Modelling Beacon Reception Probability}

Beaconing relies on broadcast transmissions in CSMA/CA. There are no retransmissions, because broadcast transmissions are not acknowledged. Furthermore, the contention window does not grow, as would occur in 802.11 unicast. This limits the choice for a backoff counter to $[0,15]$.

To model the probability of a successful beacon reception we use the probabilistic model presented by Yang et al. in [30]. In their paper they present a simplified model of the 802.11 DCF access method, used to calculate the probability of a transmission without collision.
This model is somewhat similar to slotted aloha in that time is divided into transmission slots, where the duration of one slot is equal to the time it takes to transmit a beacon, $T_{s}$. The useful lifetime of a packet, i.e., the time within which a generated beacon should be transmitted because it has otherwise been made obsolete by a freshly generated beacon, is defined as the reciprocal of the beacon generation rate: $T_{u}=\frac{1}{\lambda_{g}}$. The number of transmission slots is then given as $n_{\text {slots }}=T_{u} / T_{s}$.

The model takes into account the probability that two nodes which are within eachother's CS range may start transmitting at the same time and the probability that a hidden node may interfere with an ongoing transmission. The number of hidden nodes is set to the worst case scenario of $n / 2$, as shown in Fig. 3 . Here $n / 2$ nodes are hidden terminals for a transmission of a message from transmitter $t$ to receiver $r$.

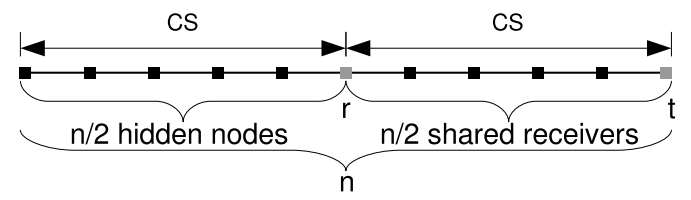

Figure 3: Worst-case hidden terminals

Assumptions with regard to vehicle topology and communication are similar to those stated in Sec. IV-A. Furthermore, because the relative distance between a transmitter and a receiver are not taken into account effects like the capture effect [40] and radio propagation are not considered.

Given all of the above assumptions the model is as follows. Denote $P_{s}$ the probability that vehicle $t$ (in Fig. 3) successfully transmits to $r$. This probability is modelled as the chance that (given a free medium and $n / 2$ shared receivers) only vehicle $t$ starts a new transmission, multiplied by the chance that no hidden nodes will interfere with this transmission: $P_{s}=P_{S R}$. $P_{H N}$. Denote $P_{a}$ as the probability that for a given slot a node attempts a transmission (if it senses medium idle): $P_{a}=$ $1 / n_{\text {slots }}=T_{s} / T_{u}$ The probability that $n / 2$ nodes will not start a transmission at the same time in that time slot, $P_{S R}$, is then given as:

$$
P_{S R}=\left(1-P_{a}\right)^{\frac{n}{2}} .
$$

Since a transmission by $t$ may experience interference from transmissions by hidden nodes that are started (i) during the time that $t$ transmits, but also (ii) in the slot previous to the slot that $t$ is transmitting in, a beacons vulnerability period is twice the beacon transmission time, making $P_{H N}$ :

$$
P_{H N}=\left(\left(1-P_{a}\right)^{\frac{n}{2}}\right)^{2}=\left(1-P_{a}\right)^{n} .
$$

Taking $P_{S R}$ and $P_{H N}$ together we get:

$$
P_{s}=\left(1-\frac{T_{s}}{T_{u}}\right)^{\frac{3 n}{2}}
$$




\section{Analysis of the Beaconing Solution Space BOUNDARIES}

As mentioned in the previous sections, the beaconing solution space extends in three dimensions; these being number of nodes, generation rate and beacon size or duration.

In order to analyze the beaconing solution space boundaries we need to identify the performance metrics that can be used to capture these boundaries. Subsequently, the beaconing solution boundaries will be projected and depicted in graphs using a numerical evaluation procedure. The numerical evaluation procedure is verified using simulations.

The performance metrics traditionally used in networking are throughput, delay and packet loss. In a beaconing application, throughput is of lesser importance than the other two. The dynamic nature of VANETs imposes requirements on delay, but these can easily be satisfied as shown by Vinel et al. in [41]. Meeting the requirements regarding the probability of successful delivery is a more problematic matter [29]. It is especially at this point that improvement is needed.

Our analysis is centered around $P_{s}$, and what can be done to maximise it in the face of varying other parameters.

Rather than specifying a required $P_{s}$, the Connect \& Drive project specifies a beacon reception rate $\lambda_{r}$. As shown in Sec. IV, $\lambda_{g}$ influences $P_{s}$ through $\mu$, and directly influences $\lambda_{r}$.

In order to provide a numerical solution from our model we use values as depicted in Table I. These values were introduced in previous sections or are realistic assumptions.

\begin{tabular}{|l|l|l|}
\hline Component & Parameter & values \\
\hline$n$ & $l$ & 4 lanes \\
& $\rho$ & $1-400 \mathrm{veh} / \mathrm{km} / 1$ \\
\hline$\lambda_{g}$ & $1-40 \mathrm{~Hz}$ & only deterministic arrivals \\
\hline$T_{s}$ & $T_{h}$ & $32+8=40 \mu \mathrm{s}$ \\
& $S$ & $100,200,400,1000,1400$ bytes \\
& $R$ & $3 \mathrm{Mbps}$ \\
& $D I F S$ & $64 \mu \mathrm{s}$ \\
& $\delta$ & $1 \mu \mathrm{s}$ \\
\hline
\end{tabular}

TABLE I.: Model parameters and values [8]

The channel utilization model was verified using the OMNet++/MiXiM simulator ${ }^{4}$. Modifications were performed to meet the assumptions made for the model; contention was eliminated, there are no hidden nodes and transmissions were perfectly aligned in time (like a slotted Time-Division Multiple Access scheme). Simulation was used to verify the bounds of the model. The results of the simulation experiments are depicted by the squares in Fig. 4. These simulation experiments show that the values that are derived analytically coincide with the simulated behavior of their distributed couterparts. This means that if the assumptions described in this paper are considered, then Eq. (1) is valid.

\section{A. Analysis of Channel Utilization Boundaries}

The model presented in Sec. IV-B describes the channel utilization. It is evident that the channel utilization of a system

\footnotetext{
${ }^{4}$ http://www.omnetpp.org, http://mixim.sourceforge.net/

${ }^{3}$ Later work will focus on shaping $\lambda_{g}$
}

can not exceed 1 (e.g. 100\% of its capacity is used). As such, if we solve Eq. (1) for $100 \%$, this will give us the maximum possible channel utilization, also known as capacity, based on the values chosen for $n, \lambda_{g}$ and $T_{s}$. Figure 4 shows a numerical solution to the model given in Eq. (1) for several values of $S$. The figure shows the channel utilization boundaries for beacon sizes stated in Table I. It should be stressed that this figure shows the maximum channel utilization, the actual achievable utilization will be considerably lower.

This deserves some clarification. Maximum channel utilization of pure ALOHA, for instance, is $18 \%$. Slotted ALOHA increases this to about 37\%, whereas Carrier-Sense Multiple Access with Collision Avoidance (CSMA/CA) used in IEEE802.11 has a typical maximum channel utilization of 54$66 \%$ in the absence of hidden terminals [42].

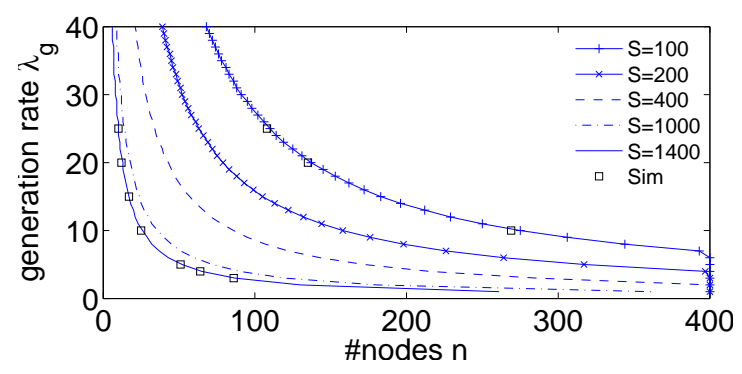

Figure 4: Channel utilization boundaries

From Fig. 4 it becomes clear that a beaconing system can have either many nodes transmitting at a low rate, or few nodes transmitting at a high rate. Furthermore, it becomes apparent that the solution space becomes larger as the beacon size decreases. It is, for instance, not possible to have 100 nodes send a beacon of 1400 bytes 10 times per second. However, it may be possible to achieve the same $n$ and $\lambda_{g}$ with a smaller beacon of 100 bytes.

\section{B. Analysis of Reception Probability}

The IEEE 802.11 DCF uses contention to avoid collisions. When the number of nodes increases, so does the probability that a collision will occur despite the collission avoidance mechanism. For this experiment we fix $\lambda_{g}$ at $25 \mathrm{~Hz}$ and $S$ at 400 bytes, conform the $C \& D$ requirements presented in Sec. III. As can be seen from Fig. 5, the probability of collision-free transmission rapidly drops as $n$ increases.

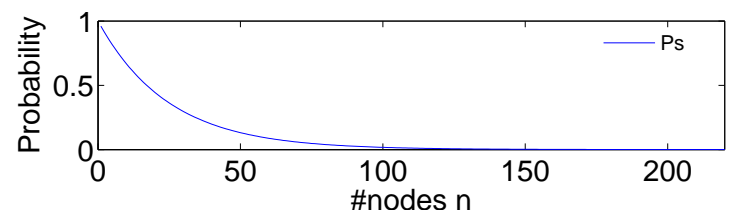

Figure 5: Probability of Collision-free transmission

Here we only consider collision-free transmission of a beacon. The impact of frame capture and multipath effects are not considered. 


\section{Beaconing Solution Space to Support C-ACC}

We can now map the C-ACC requirements from the $C \& D$ project to the derived beaconing solution space. As mentioned before, we consider beacons of 400 bytes and $\lambda_{g}=25 \mathrm{~Hz}$. If we solve Eq. (1) for these parameters we find $n=\frac{1}{25 \cdot 1.167 \mathrm{~ms}} \approx$ 34 as an upperbound for the number of vehicles within range. [24] states that a communication coverage distance of $200 \mathrm{~m} /$ 15 vehicles per lane should be considered. Consider a highway with four lanes, where $\rho=75$. In this scenario, a vehicle has 15 vehicles in the same lane up to $200 \mathrm{~m}$ ahead, but the medium has to be shared by $2 \cdot 15 \cdot 4=120$ vehicles. Clearly, $\lambda_{g}=25$ $\mathrm{Hz}$ cannot be maintained (See Fig. 4) and should, according to Eq. (1) be lowered to $\frac{1}{120 \cdot 1.167 \mathrm{~ms}} \approx 7 \mathrm{~Hz}$.

Fig. 6 shows $P_{s}$ as defined in Eq. (7) with the parameters described above. This figure supports the conclusion that 120 vehicles beaconing at $25 \mathrm{~Hz}$ will result in very low reception rates.

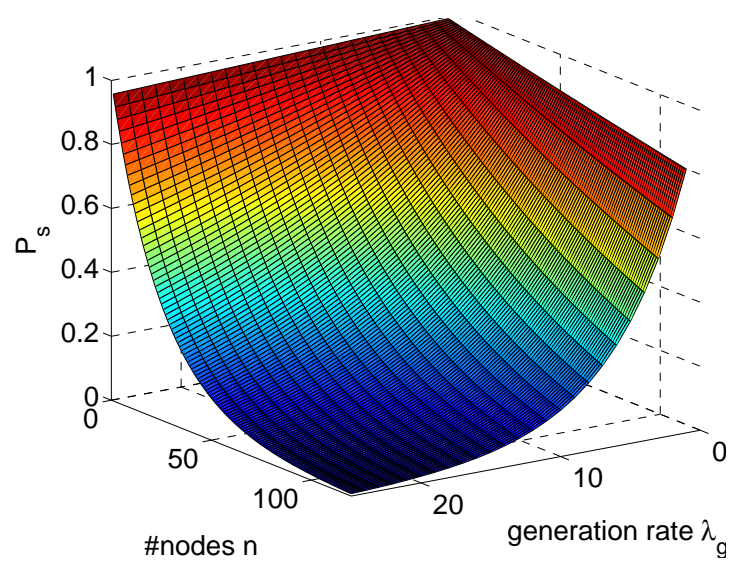

Figure 6: CACC Beaconing Solution Space

From these results, the following observations can be made:

- Since $\lambda_{r}=\lambda_{g} \cdot P_{s}$ and $P_{s}$ is determined by $\lambda_{g}$ as well as the number of nodes $n$, the beacon reception rate (the primary measure of performance in a beaconing system) can be influenced by adapting either $\lambda_{g}$ or $n$ (i.e., transmission power). The effect this has on $\lambda_{r}$ is determined by the state of the system (in terms of $n / \lambda_{g}$ ): for small $n / \lambda_{g}$, increasing either has little influence on $P_{s}$, as the medium still has enough space for increased beaconing. As a result, $\lambda_{r}$ will initially increase almost linearly with $\lambda_{g}$. For higher values of $n / \lambda_{g}$ however, increasing either may have a detrimental effect on $\lambda_{r}$ as the system is already past its saturation point. By taking the derivative of $P_{s}$ one can observe for given $n / \lambda_{g}$ whether one should either increase or decrease $\lambda_{g}$ to increase $\lambda_{r}$.

- There exists a trade-off also identified in [30]: the number of vehicles we want to collect information about, and the temporal resolution of this information. This is reflected in $n$ and $\lambda_{g}$ (or, more precisely, the resulting $\lambda_{r}$ ). Whether the beaconing system must optimize for high $n$ or $\lambda_{g}$ depends on the vehicular traffic application.
- An adaptive beaconing approach featuring both power control and generation rate looks the most promising.

In this research we assumed an omnidirectional antenna. It should be noted that C-ACC traffic information is received from ahead and transmitted behind. This gives rise to partitioning $n$ by means of antenna diversity or sectorized antennas. This practice yields significant capacity improvements in cellular communications, so application in a VANET is worth looking into.

Beaconing can provide input to many ITS applications in the safety \& efficiency domain. We reason that a single beaconing system could be designed to meet all vehicle-to-vehicle ITS application requirements and build a situational awareness from which the applications draw their input.

Neighbor knowledge is gathered in every node by means of the beaconing system; henceforth it can also be used by routing algorithms which rely on neighbor knowledge and no additional beacons of class 2 (see Sec. I) will be needed.

Beaconing alone can generate a high load on the network. Beaconing can not be simply regarded as "background traffic", because it is not (like beaconing of type 2) to support a service, it is the service. Based on our analysis we reason that a dedicated beaconing channel will greatly improve the beaconing performance and hence the performance of ITS applications using the situational awareness.

Given the requirements of ITS applications which rely on co-operative awareness such as C-ACC, any other traffic on the channel may seriously harm the beaconing performance. As a consequence the performance of safety \& efficiency applications which rely on the awareness provided by beaconing will deteriorate.

\section{CONCLUSions ANd Future WORK}

We have briefly introduced ITS applications and focused on one traffic efficiency application in particular: C-ACC. We have introduced the notion of beaconing by means of a division into three classes. Beaconing requirements have been derived from the C-ACC application and a model of channel utilization and reception probability has been proposed. The beaconing requirements were tested against the channel utilization models; the findings are summarized below.

\section{A. Conclusions}

- The beaconing solution space consists of transmission power, beacon generation rate, the size of one beacon and the data rate, the latter two of which can be grouped into message duration.

- An adaptive beaconing approach must consider control of all parameters in the solution space for maximum effect.

- Given the load generated by a beaconing system, we emphasize the need for a dedicated beaconing channel in stead of regarding beaconing as "background traffic".

- We reason a single cross-layer VANET beaconing system could fulfill requirements of beaconing at the MAC, Network and Application layer. 


\section{B. Future Work}

Future work will include design and evaluation of beaconing schemes. Field measurements are planned for proper parameterisation of propagation models with extensions to include SINR properties based on data rate and PHY properties.

Based on our findings, the $C \& D$ project will perform further experiments on the C-ACC based traffic application. These experiments will be based on simulation and prototype implementation environments. The results of these experiments will determine whether the beaconing system must be optimized for awareness or accuracy.

\section{ACKNOWLEDGEMENTS}

The authors wish to thank Alexey Vinel and Anne Remke for fruitful discussions on beaconing in VANETs, and Jeroen Ploeg and the Connect\&Drive partners for their input w.r.t. CACC. This work is sponsored by HTAS, project no. D08002.

\section{REFERENCES}

[1] "IEEE std 802.11-2007 (revision of IEEE std 802.11-1999)," pp. C1-1184, June 122007.

[2] D. Rossi, R. Fracchia, and M. Meo, "VANETs: Why use beaconing at all," pp. 2745-2751, 2008.

[3] European Telecommunications Standards Institute (ETSI), http://portal.etsi.org/its/, July 2009.

[4] International Standardization Organization (ISO) TC 204 (CALM), http://www.isotc204wg16.org, July 2009.

[5] "C2C-CC Manifesto: Overview of the C2C-CC system version 1.1," Car 2 Car Communication Consortium, Tech. Rep., 2007.

[6] T. Kosch, I. Kulp, M. Bechler, M. Strassberger, and B. Weyl, "Communication architecture for cooperative systems in europe," IEEE CM, vol. 47, no. 5, pp. 116-125, May 2009.

[7] "COMeSafety - D31 European ITS Communication Architecture," Information Society Technologies, Tech. Rep., 2008.

[8] "IEEE 802.11p draft d3.0," July 2007.

[9] I. Wilmink, G. Klunder, and B. van Arem, "Traffic flow effects of integrated full-range speed assistance (IRSA)," IEEE IV, pp. 1204-1210, 2007.

[10] B. Van Arem, C. Tampere, and K. Malone, "Modelling traffic flows with intelligent cars and intelligent roads," in IEEE IV, June 2003, pp. 456-461.

[11] P. E. Ioannou, Automated Highway Systems, 1997.

[12] D. Reichard, M. Miglietta, L. Moretti, P. Morsink, and W. Schultz, "Cartalk2000: safe and comfortable driving based upon inter-vehicle communication," in $I V, 2002$, pp. 545-550.

[13] J. Sussman, "Intelligent vehicle highway systems: challenge for the future," in Microwave Symposium Digest, 1993., IEEE MTTS International, 1993, pp. 101-104 vol.1.

[14] P. Varaiya, "Smart cars on smart roads: problems of control," Automatic Control, IEEE Transactions on, vol. 38, no. 2, pp. 195-207, Feb 1993.

[15] S. Shladover, C. Desoer, J. K. Hedrick, M. Tomizuka, J. Walrand, W. Zhang, S. H. McMahon, H. Peng, S. Sheikholeslam, and N. McKeown, "Automatic vehicle control developments in the path program," IEEE Trans. On Veh. Tech., vol. 40, pp. 114-130, 1991.

[16] A. Kanaris, P. Ioannou, and H. Fu-Sheng, "Spacing and capacity evaluations for different ahs concepts," Proc. American Control Conference, pp. 2036-2040, 1997.

[17] M. Broucke and P. Varaiya, "The automated highway system: A transportation technology for the 21st century," vol. 5, no. 11, p. 15831590, Nov 1997.

[18] R. Horowitz and P. Varaiya, "Control design of an automated highway system," vol. 88, pp. 913 - 925, July 2000.
[19] S. Tsugawa, S. Kato, K. Tokuda, T. Matsui, and H. Fujii, "An architecture for cooperative driving of automated vehicles," pp. 422-427, 2000

[20] official website of Auto 21 project, http://www.auto21.ca, July 2009.

[21] S. Halle, B. Chaib-draa, and J. Laumonier, "Car platoons simulated as a multiagent system," pp. 57-63, 2003.

[22] L. D. Baskar, B. D. Schutter, and H. Hellendoorn, "Hierarchical traffic control and management with intelliugent vehicles," pp. $834-839,2007$.

[23] Vehicle Safety Communications Project, "Final report, DOT HS 810 591," April 2006.

[24] C \& D project, Project no. HTASD08002, "C\&D WP-1 requirements document," Tech. Rep., April 2009.

[25] "Connect \& drive (C\&D), project description," http://www.ctit.utwente.nl/research/projects/national/senter/ connect-drive.doc/, July 2009.

[26] "Security in VSC-A, slides presented at IEEE 1609 meeting, August 26-28, 2008,' http://vii.path.berkely.edu/1609_wave/aug08/presentations/ VSCA-1609_080827.pdf, July 2009.

[27] "Intelligent transport systems communications access for land mobiles (calm) calm m5," ISO/TC 204, Tech. Rep.

[28] "IEEE 1609.3 trial-use standard for wireless access in vehicular environments (wave) - networking services," April 2007.

[29] A. Vinel, Y. Koucheryavy, S. Andreev, and D. Staehle, "Estimation of a successful beacon reception probability in vehicular ad-hoc networks," IWCMC, June 2009.

[30] L. Yang, J. Guo, and Y. Wu, "Channel adaptive one hop broadcasting for VANETs," in IEEE ITSC, 2008, pp. 369-374.

[31] H. T. Friis, "A note on a simple transmission formula," Proceedings of IRE, vol. 34, pp. 254-256, May 1946.

[32] M. Torrent-Moreno, "Inter-vehicle communications : Achieving safety in a distributed wireless environment : Challenges, systems and protocols," Ph.D. dissertation, 2007.

[33] "IEEE 1609 - family of standards for wireless access in vehicular environments (WAVE)," http://www.standards.its.dot.gov/, Jan 2006.

[34] W. Zhang, A. Festag, R. Baldessari, and L. Le, "Congestion control for safety messages in VANETs: Concepts and framework," in ITST, Oct. 2008, pp. 199-203.

[35] X. Chen, H. Refai, and X. Ma, "Saturation performance of ieee 802.11 broadcast scheme in ad hoc wireless lans," in IEEE VTC, 30 2007-Oct. 3 2007, pp. 1897-1901.

[36] G. Bianchi, "Performance analysis of the ieee 802.11 distributed coordination function," Selected Areas in Communications, IEEE Journal on, vol. 18, no. 3, pp. 535-547, Mar 2000.

[37] M. Torrent-Moreno, P. Santi, and H. Hartenstein, "Distributed fair transmit power adjustment for vehicular ad hoc networks," in SECON '06, vol. 2, Sept. 2006, pp. 479-488.

[38] J. Härri, F. Filali, and C. Bonnet, "Rethinking the overhead of geo-localization information for vehicular communications," in IEEE VTC, 30 2007-Oct. 3 2007, pp. 2111-2115.

[39] F. Kargl, E. Schoch, B. Wiedersheim, and T. Leinmüller, "Secure and efficient beaconing for vehicular networks," in VANET '08. ACM, 2008, pp. 82-83.

[40] J. Lee, W. Kim, S.-J. Lee, D. Jo, J. Ryu, T. Kwon, and Y. Choi, "An experimental study on the capture effect in 802.11a networks," in WinTECH '07. ACM, 2007, pp. 19-26.

[41] A. Vinel, V. Vishnevsky, and Y. Koucheryavy, "A simple analytical model for the periodic broadcasting in vehicular ad-hoc networks," in GLOBECOM Workshops, 2008 IEEE, 30 2008Dec. 4 2008, pp. 1-5.

[42] A. Athanasopoulos, E. Topalis, C. Antonopoulos, and S. Koubias, "Evaluation analysis of the performance of ieee 802.11b and ieee 802.11g standards," in ICN/ICONS/MCL, April 2006, pp. 141-141. 